\title{
Electron beam effects in high-resolution transmission electron microscopy investigations of catalytic nanoparticles
}

William Bang Lomholdt ${ }^{1}$, Matthew Helmi Leth Larsen ${ }^{1}$, Cuauhtémoc Núñez Valencia ${ }^{1}$, Jakob Schiøtz ${ }^{2}$ and Thomas Hansen ${ }^{3}$

${ }^{1}$ Technical University of Denmark, United States, ${ }^{2}$ Department of Physics, Technical University of Denmark, Kongens Lyngby, Hovedstaden, Denmark, ${ }^{3}$ DTU Nanolab, Technical University of Denmark, Kgs. Lyngby, Hovedstaden, Denmark

High-resolution transmission electron microscopy (HRTEM) is a powerful tool for atomic scale investigations of catalytic nanoparticles. The dynamics of such catalytic nanoparticles are highly dependent on the environment: temperature, reactant gases and reactor pressure. It is possible to imitate such conditions in a transmission electron microscope (TEM). Still, major gaps remain between HRTEM investigations and industrial reactors [1].

Electron beam effects play a substantial role in the interpretation of data produced in TEM investigations. There is a trade-off between optimal signal-to-noise ratio (SNR) and minimal beam damage. Furthermore, it is challenging to confirm if the beam has already caused damage prior to the signal reaching the detector [2].

In an attempt to isolate the electron beam effects from effects of the environment, we carry out a systematic study varying the beam intensity (dose rate) at different temperatures, both in high vacuum and in the presence of relevant reactant gases. The current model system consists of gold nanoparticles supported on cerium dioxide $[3,4]$. The aforementioned studies elucidate how the nanoparticles undergo changes with observation time and reactant gases present, and surface events as function of dose rate, respectively. The studies open up for further systematic parameter investigations of beam-induced surface events such as diffusion, reconstruction and damage. Furthermore, in order to assist the investigations due to high amounts of data output as well as minimizing the operator bias, artificial neural network based machine learning algorithms have been developed [5] and tested on the recorded HRTEM image sequences.

Figure 1 shows HRTEM images of two individual cerium dioxide supported gold nanoparticles at various dose rates. The SNR increases with dose rate. However, the beam intensity has significant influence on the gold nanoparticle surface characteristics. At relatively low dose rates $\left(\sim 10^{2} \mathrm{e}^{-} /\left(\AA^{2} \cdot \mathrm{s}\right)\right)$ the gold nanoparticles and support are almost indistinguishable from the vacuum background (Figure 1(a)+(b)). With dose rates at medium level $\left(\sim 10^{3} \mathrm{e}^{-} /\left(\AA^{2} \cdot \mathrm{s}\right)\right)$, the gold nanoparticle and support are easy to distinguish from the vacuum background (Figure $1(\mathrm{c})+(\mathrm{d}))$. Using high level dose rates $\left(\sim 10^{4} \mathrm{e}^{-/}\left(\AA^{2} \cdot \mathrm{s}\right)\right)$ substantially suppresses the background noise. However, two different beam-induced behaviors of the gold nanoparticles are observed: 1) Etching of the facets of the gold nanoparticle caused by electron beam sputtering (Figure 1(e), indicated by a white arrow). 2) The gold nanoparticle coalesces with neighboring gold nanoparticles (Figure 1(f), white arrows indicating gold nanoparticle crossovers). Finally, increasing the dose rates to an extremely high level $\left(\sim 10^{5} \mathrm{e}^{-} /\left(\AA^{2} \cdot \mathrm{s}\right)\right)$, the highly intense beam irreversibly damages the single gold nanoparticle (Figure $1(\mathrm{~g})$ ) and the coalesced gold nanoparticles (Figure 1(h)) by sputtering. 

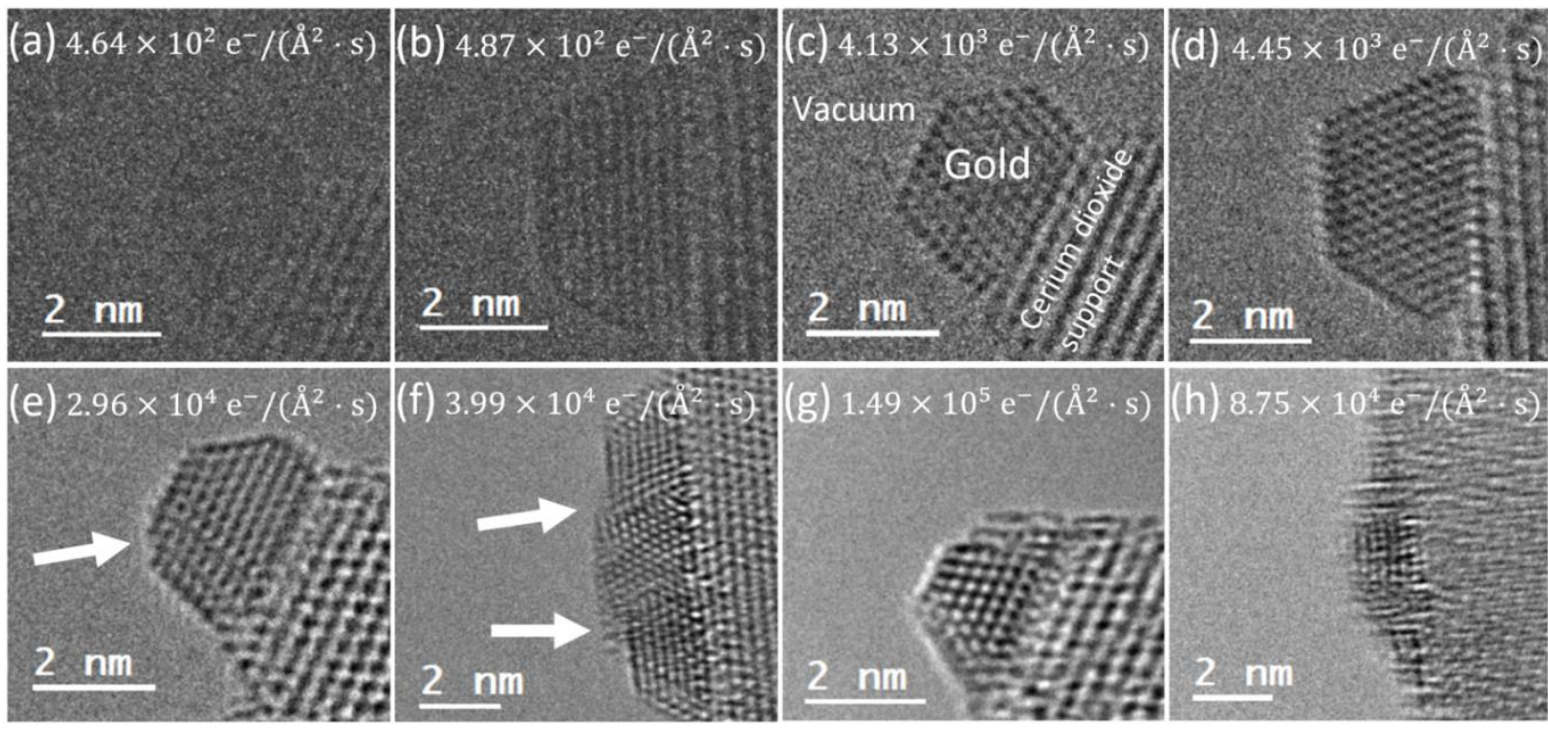

Figure 1. Effect of the increasing dose rate for cerium dioxide supported gold nanoparticles, investigated with a Titan E-cell 80-300ST TEM, at $300 \mathrm{kV}$ high-tension in high vacuum conditions, at $150{ }^{\circ} \mathrm{C}$ and at individual dose rates shown in the images. Frame exposure time is $0.2 \mathrm{~s}$, and each image shows the first frame of a video sequence. (a)+(b): Two gold nanoparticles at relatively low dose rates $\left(\sim 10^{2} \mathrm{e}^{-} /\left(\AA^{2} \cdot \mathrm{s}\right)\right)$. (c)+(d): Respectively same two gold nanoparticles as (a)+(b), at medium dose rates $\left(\sim 10^{3} \mathrm{e}^{-} /\left(\AA^{2} \cdot \mathrm{s}\right)\right)$. The gold nanoparticle, support and vacuum background are labeled in (c). (e): The gold nanoparticle in (a)+(c) at a high dose rate $\left(\sim 10^{4} \mathrm{e}^{-} /\left(\AA^{2} \cdot \mathrm{s}\right)\right)$. Etching from the electron beam is evident, indicated by the white arrow. (f): The gold nanoparticle in (b)+(d) at a high dose rate $\left(\sim 10^{4} \mathrm{e}^{-} /\left(\AA^{2} \cdot \mathrm{s}\right)\right)$, which has coalesced with neighboring gold nanoparticles. White arrows show the crossover between the individual gold nanoparticles. $(\mathrm{g})+(\mathrm{h})$ : The gold nanoparticle in $(\mathrm{a})+(\mathrm{c})+(\mathrm{e})$ and coalesced gold nanoparticles in $(\mathrm{f})$ at extremely high dose rates $\left(\sim 10^{5} \mathrm{e}^{-} /\left(\AA^{2} \cdot \mathrm{s}\right)\right)$, which both have been irreversibly damaged by sputtering from the highly intense beam.

\section{References}

[1] T. W. Hansen and J. B. Wagner, "The Structure of Catalysts Studied Using Environmental Transmission Electron Microscopy," in Controlled Atmosphere Transmission Electron Microscopy: Principles and Practice, Springer, 2016, pp. 237-258.

[2] C. Kisielowski, H. Frei, P. Specht, I. Sharp, J. Haber and S. Helveg, "Detecting structural variances of $\mathrm{Co}_{3} \mathrm{O}_{4}$ catalysts by controlling beam-induced sample alterations in the vacuum of a transmission electron microscope," Advanced Structural and Chemical Imaging, vol. 2, no. 1, p. 13, 2016.

[3] P. Liu, T. Wu, J. Madsen, J. Schiøtz, J. B. Wagner and T. W. Hansen, "Transformations of supported gold nanoparticles observed by in situ electron microscopy," Nanoscale, vol. 11, no. 24, pp. 11885-11891, 2019.

[4] P. Liu, J. Madsen, J. Schiøtz, J. B. Wagner and T. W. Hansen, "Reversible and concerted atom diffusion on supported gold nanoparticles," Journal of Physics: Materials, vol. 3, no. 2, p. 024009, 2020. [5] J. Madsen, P. Liu, J. Kling, J. B. Wagner, T. W. Hansen, O. Winther and J. Schiøtz, "A Deep Learning Approach to Identify Local Structures in Atomic-Resolution Transmission Electron Microscopy Images," Advanced Theory and Simulations, vol. 1, no. 8, p. 1800037, 2018. 